\title{
La estructura interna de la pobreza multidimensional
}

\author{
Pablo Beytía ${ }^{1}$
}

(Última versión de autor)

\begin{abstract}
Capítulo incluido en: Catalina Siles (ed.). 2016. Los Invisibles. Por qué la pobreza y la exclusión social dejaron de ser prioridad. Santiago de Chile: Instituto de Estudios de la Sociedad, pp. 71-88.
\end{abstract}

\section{Introducción}

Las políticas públicas que buscan la superación de la pobreza necesitan un acuerdo inicial sobre qué considerar oficialmente como pobreza. Ello permite identificar a quiénes se encuentran en dicha situación, definir la magnitud social del fenómeno, entender sus causas y consecuencias, diseñar soluciones apropiadas y evaluar la eficacia de los programas o medidas sociales que se desarrollen al respecto. En su versión primaria, el problema político que se impone es el siguiente: ¿a quién debe dirigirse una política pública que busca superar la pobreza? Y la respuesta parece simple: hacia «el pobre». Pero, ¿quién es «el pobre»? ¿Cuáles son las características que lo distinguen de otros ciudadanos? Esta es la importante respuesta que nos entregan los indicadores de pobreza ${ }^{2}$.

La creación de dichos indicadores es indispensable para una adecuada aplicación de las políticas sociales. Sin embargo, ellos deben ser seleccionados con extremo cuidado, porque la información que ofrecen genera consecuencias sociales en la población que se intenta describir. Cada indicador funciona como un par de lentes ópticos con un filtro determinado: visibiliza una realidad, al costo de esconder todo lo demás. Si definimos, por ejemplo, la pobreza según los ingresos de cada hogar, no observaremos las condiciones de trabajo, vivienda, salud y educación, y ello tiene efectos importantes en nuestra comprensión de la situación social y en las propuestas de políticas públicas que podamos diseñar al respecto. Los indicadores sociales son, entonces, simplificaciones con efectos políticos y ciudadanos. Ellos encasillan a las personas en categorías, lo cual frecuentemente tiene consecuencias en su posterior acceso a recursos públicos. Según el indicador de referencia, se identificarán distintas personas como pobres, se apreciarán diferentes magnitudes de pobreza en la población y se crearán diversas estrategias para abordarla. El tipo

\footnotetext{
1 Profesor de la cátedra «Pobreza y segregación urbana», en la Pontificia Universidad Católica de Chile. Director del Centro de Investigación Social de TECHO-Chile entre los años 2013 y 2014. Sociólogo y Magíster en Sociología, Pontificia Universidad Católica de Chile, y Magíster en Filosofía por la Universidad de Chile.

2 Esta es una tarea conceptualmente compleja, dadas la múltiples definiciones de «pobreza». Sobre esto, véase: Paul Spicker "Definiciones de pobreza: doce grupos de significados", en Pobreza: un glosario internacional, Eds. Paul Spicker, Sonia Álvarez y David Gordon (Buenos Aires: Consejo Latinoamericano de Ciencias Sociales - CLACSO, 2009).
} 
de indicador suele condicionar, finalmente, el nivel de atención y sensación de urgencia que presenta la pobreza en un contexto social específico.

Por todas estas razones, no es trivial que un país entre en un proceso de reflexión sobre sus indicadores oficiales de pobreza. Tal ha sido el caso de Chile en los últimos años. El interés político por actualizar y complementar la medición se inició oficialmente en diciembre de 2012, cuando fue constituida la "Comisión Asesora Presidencial de Expertos para la Actualización de la Línea de la Pobreza y la Pobreza Extrema". Durante trece meses, esta delegación desarrolló un proceso participativo, en el cual trabajaron diez especialistas con el apoyo de un centenar de personas y decenas de instituciones. El resultado fue un informe publicado en enero de 2014, en donde se hicieron dos grandes propuestas: a) una manera de actualizar la medición de pobreza por ingresos - la cual se venía aplicando en Chile desde el año 1987- y b) una forma multidimensional de medir la pobreza ${ }^{3}$. Ambas ideas fueron recibidas por el Ministerio de Desarrollo Social, que durante un año elaboró nuevas metodologías oficiales para la medición de la pobreza, publicadas en enero de 20154. En ese mismo mes, se dieron a conocer los resultados de la Encuesta de Caracterización Socioeconómica Nacional (CASEN) efectuada el año 2013, aplicando estas nuevas metodologías de análisis a la muestra nacional ${ }^{5}$.

El mayor cambio realizado en este proceso fue la inclusión de un indicador oficial de pobreza multidimensional, que se construye a partir de doce variables distribuidas en cuatro dimensiones - educación, salud, trabajo y seguridad social, y vivienda- Tradicionalmente el Estado chileno había entendido la pobreza únicamente a través del nivel de ingreso per cápita del hogar, lo cual en el mejor de los casos era una aproximación indirecta a otras múltiples expresiones del fenómeno. Ahora, en cambio, se dio el paso de observar directamente las carencias en los cuatro ámbitos señalados, entendiendo que la pobreza es un fenómeno complejo, con manifestaciones y soluciones diversas. Si hasta agosto de 2012, el Ministro de Desarrollo Social podía argumentar públicamente que sólo existen dos elementos claves para entender el aumento y la disminución de la pobreza en Chile - el precio de los alimentos y el nivel de empleo-6, hoy esa frase parecería simplemente absurda. Es necesario entender también las dinámicas educativas, sanitarias y habitacionales, e incluso observar la composición de los vínculos sociales y del entorno urbano ${ }^{7}$.

3 Comisión para la Medición de la Pobreza, Informe Final (2014). Disponible en: http://www.cl.undp.org/content/dam/chile/docs/pobreza/undp_cl_pobreza_comision_pobreza_2 014.pdf (consultado en mayo de 2015).

${ }^{4}$ Ministerio de Desarrollo Social, "Nueva metodología de medición de la pobreza por ingresos y multidimensional", Serie de Documentos Metodológicos, 28 (2015).

${ }_{5}$ Ministerio de Desarrollo Social, "Situación de la pobreza en Chile. Presentación de la nueva metodología de medición de la pobreza y síntesis de los principales resultados". Disponible en:

http://observatorio.ministeriodesarrollosocial.gob.cl/documentos/Casen2013_Situacion_Pobreza_ Chile.pdf (consultado en mayo de 2015).

${ }^{6}$ Exposición de Joaquín Lavín, entonces Ministro de Desarrollo Social, el 8 de agosto de 2012 en el Centro de Estudios Públicos. El video del seminario y sus diapositivas se encuentran disponibles en: http://www.cepchile.cl/1_5104/doc/videos_seminario_la_encuesta_casen.html\#.VVn_-Np_Okp

7 Según ha declarado el Ministerio de Desarrollo Social, el proyecto de la medición multidimensional de la pobreza se completará en la próxima encuesta CASEN, al incluir una quinta dimensión sobre el entorno y las redes sociales. 
Ahora bien: la generación de un indicador multidimensional no sólo permite entender la pobreza en un nivel mayor de complejidad, sino que también abre la puerta a un nuevo tipo de análisis, centrado en observar las relaciones entre las distintas variables que componen la pobreza multidimensional. En otras palabras, desde que existe en Chile un indicador complejo (con múltiples variables) es posible analizar la estructura interna de la pobreza, algo que hasta el momento no ha sido efectuado sistemáticamente en ningún estudio. Imaginemos, por un momento, que el indicador de pobreza se compone de doce variables primarias y que ellas se relacionan entre sí en diverso grado. Si pudiéramos graficar estas relaciones - por ejemplo, con una línea más débil o gruesa según la fuerza del vínculo-, veríamos que entre las doce variables se establece una especie de red de relaciones, en donde algunas variables son más centrales que otras. A mi juicio, este entendimiento reticular de la pobreza ofrece buenas pistas sobre las dinámicas internas del fenómeno, y ello podría ayudar en la orientación de las políticas públicas.

El objetivo de este artículo es analizar la estructura interna de la pobreza multidimensional en Chile, entendiendo que este fenómeno funciona como una red de variables vinculadas entre sí, y que dicha red presenta propiedades que no serían observadas al analizar los indicadores por separado o sumados entre sí. Para ello, presentaré en primer lugar una metodología que permite observar esta estructura interna. Luego mostraré los principales resultados de este tipo de análisis. Finalmente, expondré las conclusiones que se pueden extraer de estos datos, tanto sobre el concepto de pobreza multidimensional como sobre la manifestación de la pobreza en Chile.

\section{Desde la «suma de indicadores» hacia la «red de relaciones»: una metodología para estudiar la estructura interna de la pobreza}

La medición multidimensional de la pobreza consiste en evaluar si las personas logran superar umbrales mínimos en diferentes dimensiones del bienestar individual $^{8}$. Es un enfoque que se ha popularizado en el último tiempo, tanto por los escritos conceptuales del premio Nobel de economía Amartya Sen ${ }^{9}$, como por la creciente disponibilidad de datos que permiten describir en detalle a las poblaciones vulnerables. Actualmente existen diversas metodologías para elaborar indicadores multidimensionales. En el caso de Chile, se utilizó como base la propuesta metodológica de Sabina Alkire y James Foster, desarrollada en 2007 en el marco de la Iniciativa de Pobreza y Desarrollo Humano de la Universidad de

\footnotetext{
8 François Bourguignon y Satya Chakravarty, "The measurement of multidimensional Poverty", Journal of Economic Inequality, 1 (Abril 2003). También: CEPAL, "La medición multidimensional de la pobreza" (Pucón: CEPAL, 2013), 4.

9 Algunos escritos de Amartya Sen que apuntan en esta dirección son: "Poverty: An Ordinal Approach to Measurement", Econometrica, № 44 (1976); "Well-Being, Agency and Freedom: The Dewey Lectures 1984", The Journal of Philosophy, № 82 (1985); The Standard of Living (Cambridge: Cambridge University Press, 1987); "Capability and Well-Being", en: Marta Nussbaum y Amartya Sen (Eds.), Quality of Life (Oxford: Clarendon Press, 1993); Desarrollo y libertad (México, D. F.: Planeta, 2000).
} 
Oxford ${ }^{10}$. Este modelo fue utilizado previamente por algunos países de América Latina - como México y Colombia- y por las Naciones Unidas en su confección del Î́ndice de Pobreza Multidimensional.

La metodología específica de Chile, utiliza a los hogares como unidad de análisis. Los individuos serán pobres según la situación de vida que tengan sus hogares en cuatro ámbitos: a) educación, b) salud, c) trabajo y seguridad social, y d) vivienda. Cada una de estas dimensiones está medida por tres indicadores, sumando un total de doce variables primarias, todas ellas disponibles en la encuesta CASEN. La organización interna de cada dimensión, incluyendo los indicadores específicos que la componen, puede observarse en el siguiente esquema:

\section{Dimensiones e indicadores primarios de pobreza multidimensional ${ }^{11}$}
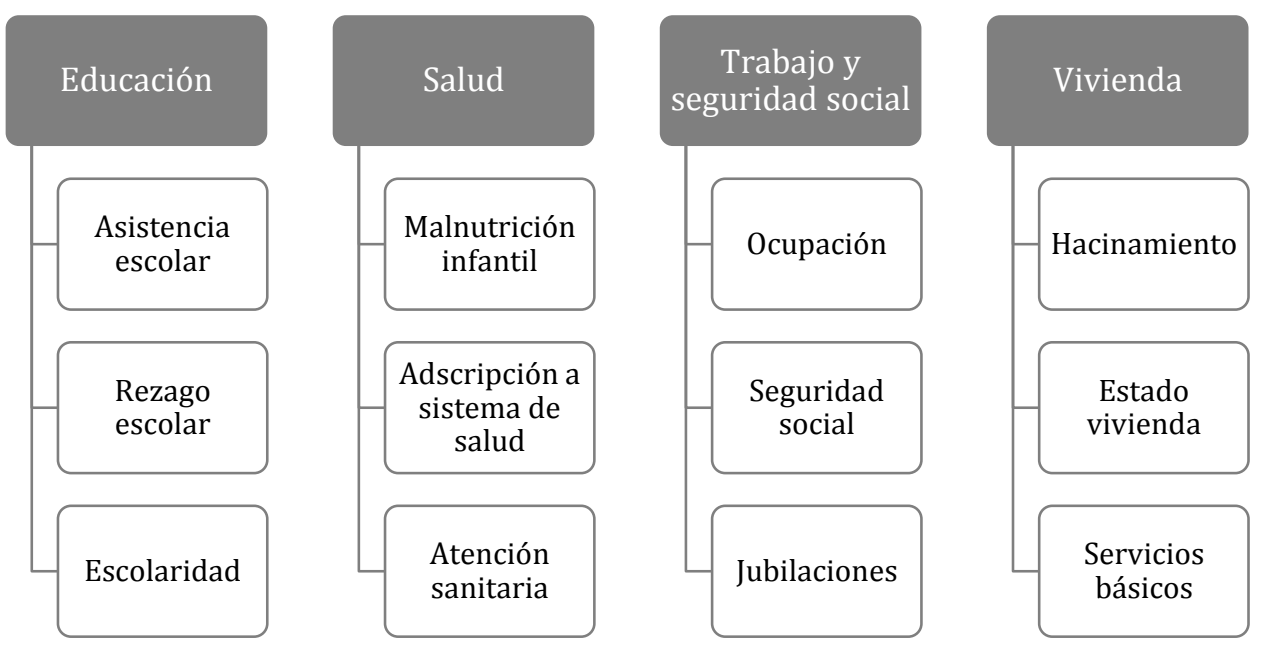

Cada una de estas doce variables primarias posee un umbral específico, a partir del cual se determina si existe o no una privación ${ }^{12}$. Todas las privaciones se suman entre sí con igual ponderación, para elaborar el indicador general de pobreza multidimensional. Una vez sumado este indicador, se considera que un hogar está en situación de pobreza si tiene al menos un $25 \%$ del total de privaciones posibles (tres variables que no superen su umbral específico), lo que es equivalente a una dimensión completa ${ }^{13}$.

Para el desarrollo de este estudio, se replicaron con exactitud las doce variables con sus umbrales específicos, utilizando la encuesta CASEN 2013 y siguiendo la programación de indicadores documentada por el Ministerio de Desarrollo Social ${ }^{14}$. Pero en vez de sumar los indicadores - tal cual se procede en la generación del indicador multidimensional-, se calcularon las correlaciones entre todas estas

\footnotetext{
10 Sabina Alkire y James Foster, “Counting and Multidimensional Poverty Measurement”, OPHI Working Paper, № 7 (2007).

11 Ministerio de Desarrollo Social, "Nueva metodología de medición de la pobreza por ingresos y multidimensional”, Serie de Documentos Metodológicos, № 28 (2015), 29.

12 Cada uno de los umbrales es explicado en: Íbidem.

13 Íbidem, 45.

14 Ministerio de Desarrollo Social, "Pobreza Multidimensional: anexo programación de indicadores y medición”, Serie de Documentos Metodológicos, № 28 (2015), 29.
} 
variables $^{15}$. Con esta información, se prepararon los datos en una matriz de doble entrada, para ser analizados como una red de correlaciones. En esta red se incluyeron las correlaciones entre variables que cumplían con dos requisitos: a) tener un vínculo estadísticamente significativo ${ }^{16} \mathrm{y}$ b) que éste vínculo sea positivo -es decir, que la carencia en una variable esté vinculada estadísticamente con la carencia en otra variable-17.

Los datos de estos vínculos se analizaron en un programa computacional de análisis de redes sociales (Agna), que permitió graficar la red de correlaciones y observar los patrones estructurales que en ella se desarrollan. Con este programa, cada vínculo significativo entre dos variables se graficó con una línea que las une, y que aumenta su grosor mientras más fuerte es el vínculo entre dichas variables. Además, se calcularon distintos coeficientes que permiten indagar en el peso estructural que tiene cada una de las doce variables incorporadas en el indicador de pobreza multidimensional.

Finalmente, se sumaron las tres variables (privaciones) que componen cada dimensión, para formar índices de pobreza dimensionales. Ello permitió calcular las correlaciones entre las cuatro dimensiones de pobreza, formando una segunda red de análisis. En esta segunda red, se calculó también el peso estructural y el porcentaje de variación mutua que tiene cada dimensión con las otras restantes ${ }^{18}$.

\section{La pobreza como red multidimensional de privaciones}

Como se ha mencionado, los indicadores de pobreza multidimensional se caracterizan por incluir varias dimensiones de análisis, cada una de ellas compuesta a su vez por distintas variables, donde cada variable representa un tipo de privación posible. De esa manera se configura la medición oficial en Chile, que se compone de cuatro dimensiones, todas ellas integradas por tres variables primarias, sumando un conjunto de doce carencias posibles. Esta nueva medición se ha entendido como una oportunidad para expandir el nivel de complejidad en que actualmente entendemos la pobreza y para observar directamente algunos fenómenos de privación que antes quedaban velados 0 aproximados indirectamente por medio del ingreso.

Esta nueva forma de medición, no obstante, también permite el entendimiento de la estructura interna de la pobreza: observar las relaciones entre los doce indicadores

\footnotetext{
${ }^{15}$ La correlación entre cada par de variables fue medida con el coeficiente estadístico de Pearson, que mide el nivel de relación entre dos variables cuantitativas. El mínimo de relación es representado por un coeficiente de 0 , mientras que el máximo tendría un coeficiente de 1 (cuando la relación es positiva) 0 -1 (cuando la relación es negativa).

${ }^{16} \mathrm{~A}$ un nivel de significancia de 0,01 (bilateral).

17 No se consideraron las relaciones negativas entre variables, dado que ellas no implicarían un aumento o disminución en el indicador general de pobreza multidimensional: si la carencia de una variable se relaciona con la no carencia de otra, no hay un cambio en el escenario global de indicadores - no hay mayor ni menor pobreza multidimensional-, porque es un juego de suma cero.

18 Este último porcentaje se obtiene calculando el 'coeficiente de determinación' de cada correlación.
} 
primarios y las propiedades estructurales que surgen en esta red de correlaciones. Dado que normalmente las privaciones no funcionan de manera aislada, sino que se vinculan entre sí en manifestaciones coordinadas, es importante observar la complejidad interna de este fenómeno. Con esta orientación, la pobreza multidimensional no se entenderá simplemente como una suma de privaciones, sino que como un fenómeno reticular con dinámicas internas y propiedades que emergen al observar la red completa de relaciones. En otras palabras, la pobreza sería más que la suma de sus indicadores, ya que en la estructura relacional de las distintas privaciones surgen sinérgicamente propiedades no observadas con la simple separación o agregación de indicadores. Como puede notarse, esta forma de observar la pobreza se caracteriza por un enfoque holista, que contrasta con la visión analítica tradicionalmente utilizada en los estudios sobre pobreza.

El primer paso para entender la estructura interna de la pobreza multidimensional de Chile, es graficar las correlaciones entre los diversos tipos de privaciones que la componen. El gráfico 1, muestra los vínculos directos y estadísticamente significativos entre los doce tipos de carencias que se agregan en el indicador de pobreza multidimensional:

\section{Gráfico 1. Red de correlaciones entre privaciones}

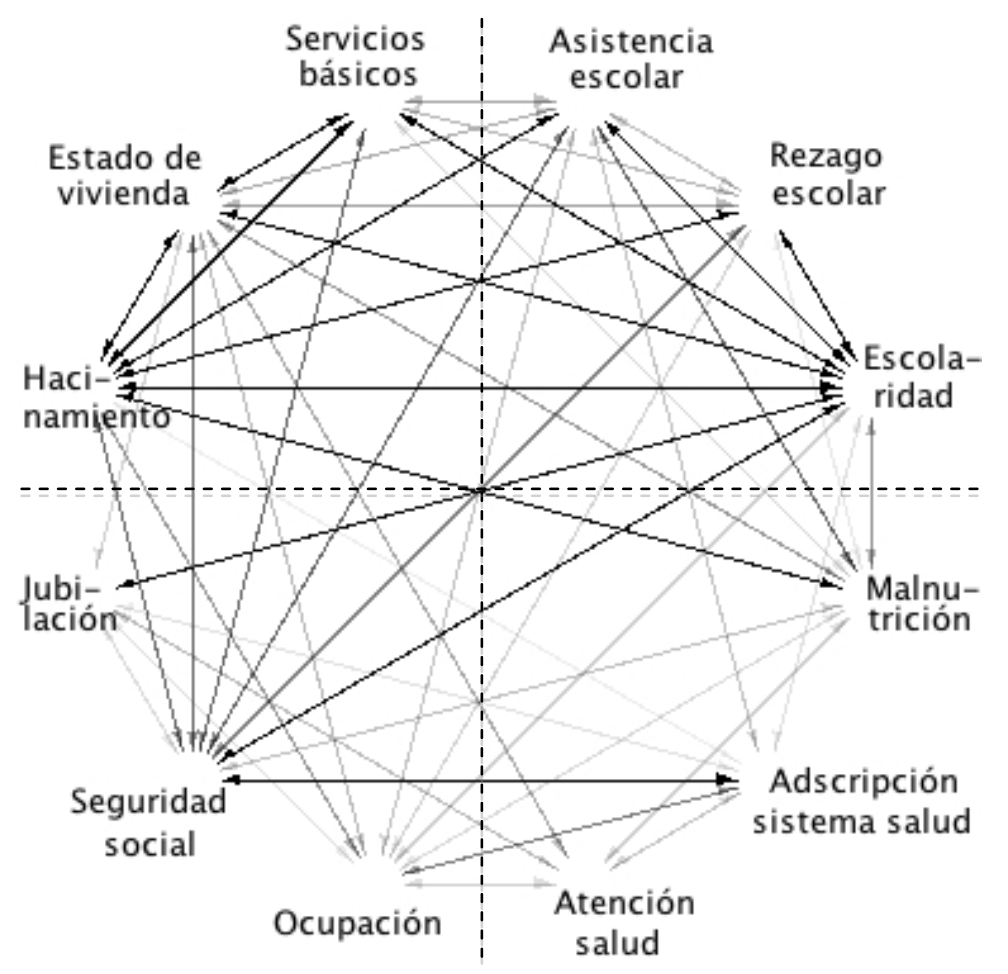

Las variables de este gráfico están ordenadas según las cuatro dimensiones que comprenden la medición de pobreza multidimensional: en el extremo superior izquierdo se encuentran los indicadores de vivienda, en el superior derecho los de educación, en el inferior izquierdo los de trabajo y seguridad social, y en el inferior derecho los de salud. Mientras más gruesa es la línea que une dos variables, mayor es la correlación entre dichas variables. Dicho de otra forma: mientras más marcado se muestra un vínculo en el gráfico, es más común que los dos tipos de privaciones se manifiesten al mismo tiempo en los hogares del país. 
Al observar gráficamente esta red de privaciones, inmediatamente se entiende que hay variables que estructuralmente tienen mayor peso que otras. Esto no es un dato menor: la medición oficial de pobreza en Chile, con su método de sumar carencias, pretende igualar el peso que tiene cada variable en el indicador general de pobreza. Sin embargo, la observación de las relaciones entre dichos indicadores muestra que las variables no son equivalentes al momento de manifestarse en los hogares: es muy diferente, por ejemplo, tener una deficiente atención en salud a tener privación de escolaridad, porque esta segunda carencia tiene un vínculo mucho mayor con otras manifestaciones de pobreza. Los hogares que están privados de niveles básicos de escolaridad, suelen estar acompañados por otras privaciones, algo que es menos común entre quienes carecen de una atención sanitaria mínima.

Para medir el peso estructural de cada variable de pobreza se calcularon los «grados nodales» de todos los indicadores. Siguiendo la terminología de análisis de redes, los «nodos» son los elementos incorporados en la red —en este caso, los doce indicadores de pobreza-. Sucesivamente, el grado nodal sería un coeficiente que suma el total de relaciones de cada nodo, ponderándolas según la fuerza de dicha relación. El resultado, entonces, es más alto mientras más relaciones tenga un indicador con otras variables y mientras más fuertes sean dichas relaciones, siendo una excelente aproximación al peso estructural de cada variable en la conformación de la red de privaciones. El gráfico 2, muestra el grado nodal de cada indicador en la red multidimensional de pobreza:

\section{Gráfico 2: Peso estructural de los indicadores de pobreza, según su grado nodal}

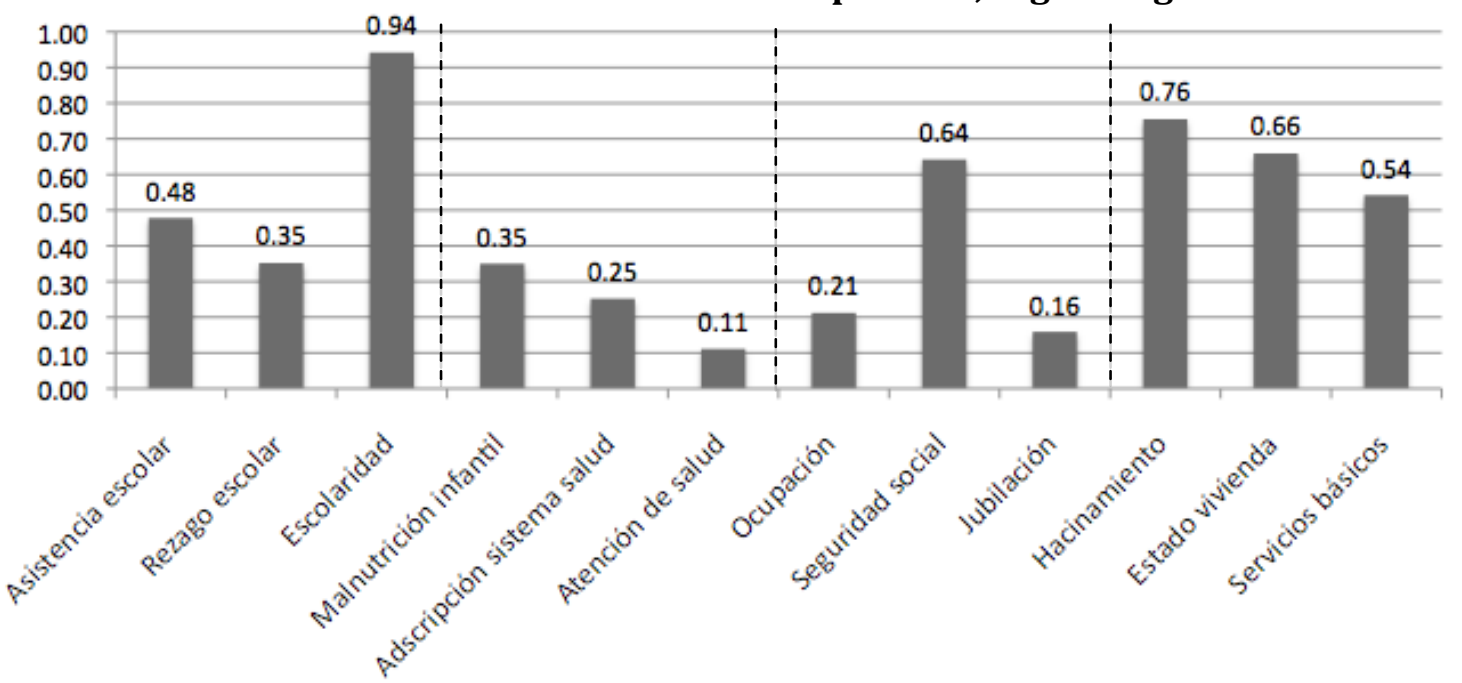

Como puede observarse, son cinco las variables que tienen mayor peso estructural en la red de relaciones (en orden de mayor a menor peso): escolaridad, hacinamiento, estado de la vivienda, seguridad social y servicios básicos. Se trata de tres variables incluidas en la dimensión de vivienda, una de educación y otra de trabajo y seguridad social. La privación de escolaridad en un hogar, desde esta perspectiva estructural, sería el mejor predictor simple de que en ese hogar existe pobreza multidimensional, porque es el indicador que se relaciona en mayor grado con otras carencias dentro de esta red. En contraste, la dimensión de salud es 
aquella que posee indicadores con menor peso estructural, ya que sus privaciones están poco vinculadas con otras privaciones.

Para evaluar más completamente la importancia de cada variable en la manifestación conjunta de distintas privaciones, también es necesario evaluar su papel como «nodo intermediador». Por ejemplo, la existencia de malnutrición en un hogar no está estadísticamente vinculada con la privación de una jubilación adecuada; sin embargo, ella sí se relaciona con la carencia de escolaridad en el hogar, y ésta última se vincula con la privación en términos de jubilación. En este ejemplo, la carencia de escolaridad actuaría como «intermediador», ya que es el enlace que permite reunir en un mismo hogar otras dos privaciones que no están vinculadas directamente entre sí. Cada actuación de intermediación, entonces, permite que se manifiesten tres carencias en un mismo hogar, lo cual sería equivalente a que las personas de ese hogar sean consideradas como pobres multidimensionalmente. El gráfico 3 muestra el grado de intermediación ${ }^{19}$ que tiene cada indicador en la red de pobreza:

\section{Gráfico 3: Grado de intermediación de los indicadores de pobreza}

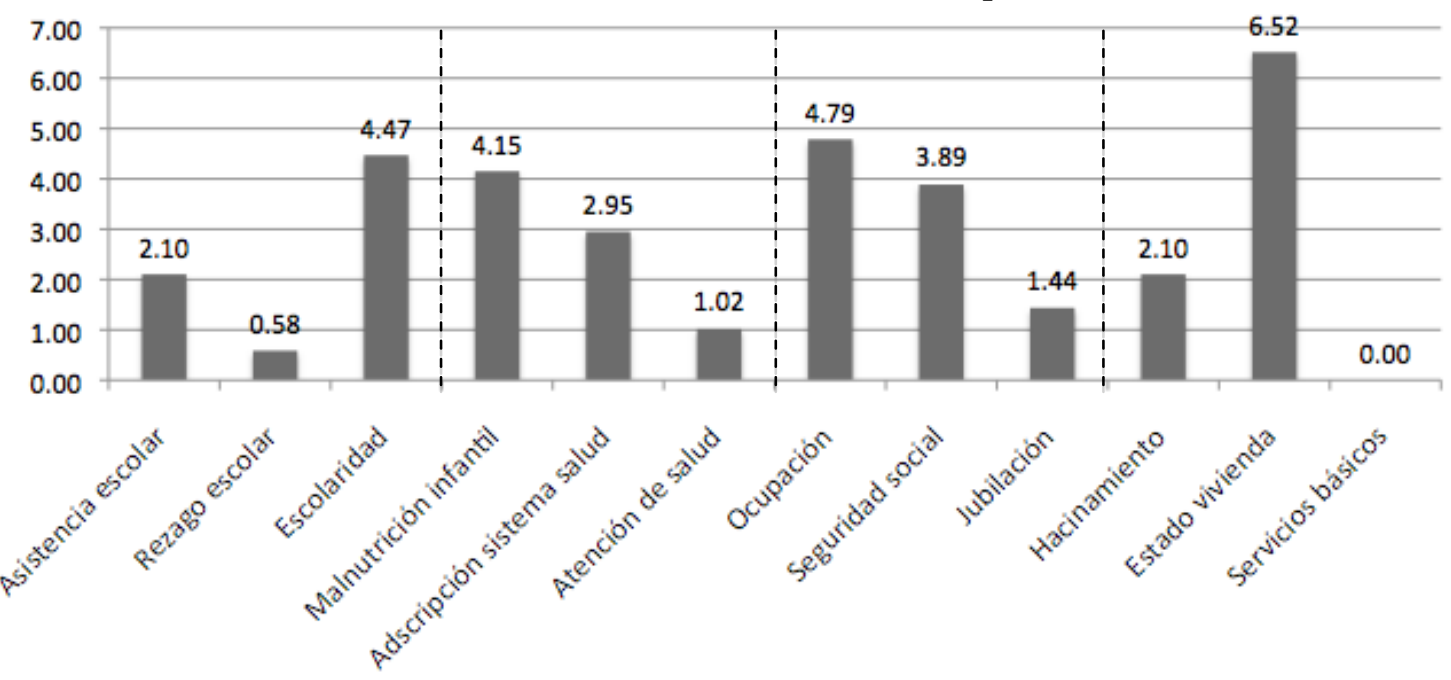

Como puede notarse, el déficit en el estado de la vivienda es el mayor intermediador entre carencias que no se relacionan directamente, lo que lo convierte en un indicador clave para comprender la dinámica interna de la pobreza multidimensional. Por ejemplo, la privación de atención en salud no se relaciona significativamente con la carencia en ningún aspecto educativo -asistencia, rezago o escolaridad-, ni con el hacinamiento o las privaciones de seguridad social y de servicios básicos en el hogar. Sin embargo, su principal vínculo es con el mal estado de la vivienda, y éste sí se vincula significativamente con los seis indicadores recién señalados. Por lo tanto, la carencia de atención sanitaria tiende a manifestarse junto a esas otras privaciones cuando existe también una carencia en el estado de la vivienda. Muchas veces hay más de un intermediador posible entre dos privaciones no relacionadas directamente; no obstante, al sumar todas las intermediaciones, el estado de la vivienda aparece como el indicador que permite reunir a más privaciones y de la manera más directa.

${ }^{19}$ El coeficiente de intermediación expresa la cantidad de "rutas mínimas" (o distancia geodésica) en que cada variable funciona como intermediadora para que se relacionen dos variables entre sí. 
Por otra parte, la red de pobreza multidimensional puede ser analizada en un segundo nivel de abstracción. Hasta el momento hemos observado las relaciones entre las doce privaciones que componen la medición general de pobreza. No obstante, estas carencias están organizadas en cuatro dimensiones -educación, salud, trabajo y vivienda-, que muestran diferentes niveles de centralidad en el fenómeno global. Para entender la estructura de relaciones entre estas dimensiones de pobreza, se formaron índices que suman la cantidad de privaciones que tienen los hogares en cada dimensión. Luego se calcularon las correlaciones entre estos índices dimensionales de pobreza. El gráfico 4 muestra los vínculos directos y estadísticamente significativos entre las cuatro dimensiones de pobreza consideradas en el indicador multidimensional:

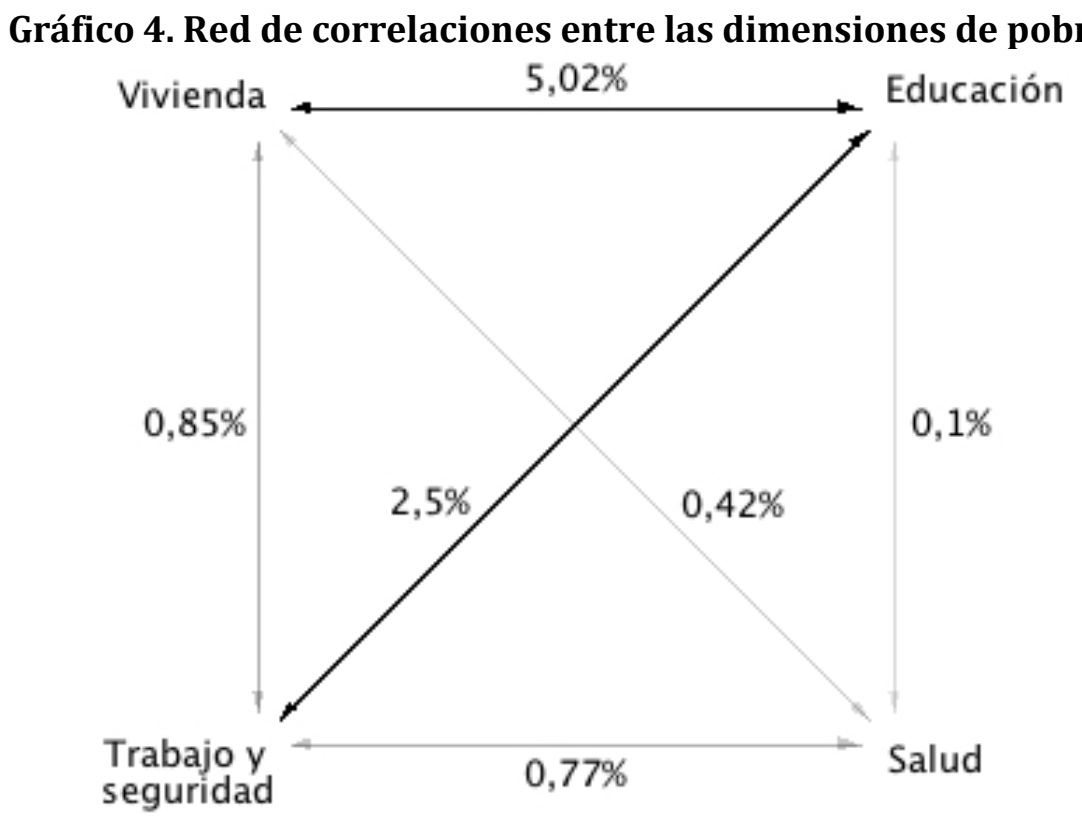

En este caso, cada línea va acompañada con un número que expresa el porcentaje de variación conjunta entre las dimensiones de pobreza vinculadas ${ }^{20}$. Por ejemplo, las carencias de vivienda se relacionan un 5,02\% con las privaciones de educación, siendo éstas las dimensiones mayormente vinculadas en la red de pobreza multidimensional. La privación educacional también se correlaciona en gran medida con la pobreza de trabajo y seguridad social $(2,5 \%)$. En contraste, la dimensión de salud parecer ser la más desconectada de la red, teniendo una escasa variación conjunta con la educación $(0,1 \%)$, la vivienda $(0,42 \%)$ y el trabajo $(0,77 \%)$.

Para comprobar el peso estructural de cada dimensión en la red de pobreza multidimensional, se utilizó el mismo procedimiento efectuado con los doce indicadores de pobreza: se calculó el «grado nodal» de cada dimensión, es decir, aquel coeficiente que suma el total de relaciones de cada ámbito de la red,

20 Este número es el "coeficiente de determinación”, indicador estadístico que permite expresar el porcentaje de correlación entre dos variables. 
ponderándolas según la fuerza del vínculo. El resultado de este ejercicio puede observarse en el gráfico 5 :

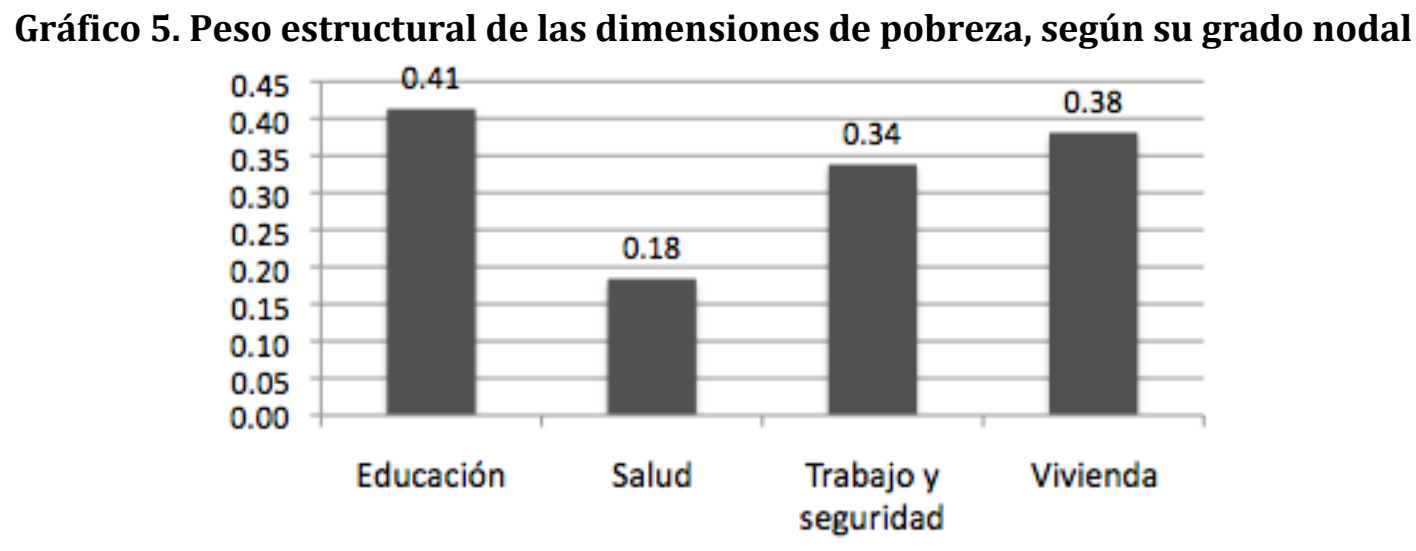

Como puede notarse, la educación es la dimensión con mayor peso estructural en la red de pobreza multidimensional, seguida de cerca por las dimensiones de vivienda y de trabajo y seguridad. Llama la atención que las carencias sanitarias sean notoriamente aquellas que están menos conectadas con otros tipos de privaciones: eso significa que tienen una menor centralidad en la estructura multidimensional de la pobreza chilena.

\section{Nuevas pistas sobre la pobreza multidimensional y el caso chileno}

El ejercicio efectuado en este artículo muestra el potencial de desarrollar un método complementario para el análisis de la pobreza multidimensional en Chile. A mi juicio, no sólo es importante tener un foco analítico - que muestra información separada para cada indicador y dimensión incorporados en la medición-, sino también un enfoque relacional, que observe los vínculos entre los diferentes tipos de privaciones. Con este método, la pobreza no se entiende únicamente como un fenómeno agregado, sino también como una estructura compleja, con un tipo específico de organización interna.

El paso efectuado desde un enfoque analítico-agregado a uno relacionalestructural permite extraer algunas conclusiones generales sobre el estudio de la pobreza multidimensional. En primer lugar, que ella debe ser tratada como un fenómeno con propiedades emergentes, las cuales surgen sinérgicamente al analizar la estructura de relaciones entre privaciones. Elementos como el peso estructural o la intermediación, son una parte constituyente del fenómeno estudiado, a pesar de que no hayan sido visibilizados en las investigaciones precedentes. En ese sentido, debe entenderse que la pobreza multidimensional es más que la suma de sus indicadores, aunque la observación de estas propiedades necesite el despliegue de un análisis diferente al que tradicionalmente se ha efectuado.

En segundo lugar, del análisis efectuado se desprende que los indicadores de pobreza multidimensional no suelen ser equivalentes entre sí para la comprensión y explicación de la pobreza, a pesar de que sean ponderados con el mismo peso en el 
indicador agregado. Ello porque es muy difícil que tengan el mismo peso estructural, si se observa la red de relaciones entre los distintos tipos de privaciones. En otras palabras, el análisis relacional-estructural muestra que para la comprensión y explicación de la pobreza multidimensional existen algunas carencias y dimensiones de carencias más importantes que otras; ello sucede a pesar de que conceptualmente entendamos que todos los indicadores y dimensiones son igualmente importantes para que alguien sea considerado como pobre.

Por otra parte, los datos analizados permiten extraer algunas conclusiones sobre la situación actual de la pobreza en Chile. La más relevante, es que existe una mayor importancia estructural de dos dimensiones: a) la educación -especialmente por el peso y la intermediación del nivel de escolaridad-y b) la vivienda - en donde el hacinamiento, los servicios básicos y el estado de la vivienda destacan por su peso estructural, teniendo esta última variable además un importante rol como agrupadora de privaciones en un mismo hogar- Este resultado no debe interpretarse de una manera causal, en el sentido de que la educación y la vivienda expliquen en mayor medida que las personas entren o salgan de la pobreza ${ }^{21}$. Sin embargo, la evidencia muestra que ambas dimensiones tienen una mayor relevancia para entender cómo se expresa multidimensionalmente la pobreza en Chile, especialmente si se busca comprender la organización interna del fenómeno y reconocer qué privaciones tienden a manifestarse conjuntamente en los hogares.

Por otra parte, los resultados de este estudio podrían utilizarse para establecer prioridades en la investigación sobre la pobreza chilena. Sería deseable que futuras investigaciones sobre las causas de la pobreza pongan especial énfasis en el efecto de las privaciones que tienen mayor centralidad en la red de pobreza multidimensional -escolaridad, hacinamiento, estado de la vivienda, seguridad social y servicios básicos-. Estas cinco carencias tienden a manifestarse en los hogares agrupando múltiples privaciones; por ello, si además tuvieran un alto peso en la explicación causal del fenómeno, podrían concebirse como el núcleo o centro de gravedad de la pobreza chilena.

Finalmente, la información de este estudio también podría ser utilizada para mejorar la focalización de las políticas sociales. El modelo de pobreza multidimensional tiene enormes virtudes, pero su impronta multifocal también podría confundir a los formuladores de políticas públicas. Dado que ellos cuentan con recursos limitados, constantemente deben priorizar la intervención de algunas privaciones presentadas en la población. Los datos estructurales de esta investigación pueden ser útiles para focalizar los esfuerzos y recursos, dado que sería deseable priorizar la intervención de aquellas carencias que se vinculan en mayor medida con la manifestación de otras carencias.

Sin embargo, el criterio estructural no debería ser el único utilizado en las políticas sociales, dado que - al menos como ha sido trabajado en este artículo- ofrece una visión sobre la manifestación de la pobreza actual de Chile, pero no sobre aquello que explica el surgimiento de ella misma. En ese sentido, sería apropiado que las

${ }^{21}$ Para ello se necesitaría desarrollar un estudio longitudinal, con seguimiento de hogares a través del tiempo. 
políticas que busquen erradicar la pobreza focalicen sus recursos ponderando al menos tres criterios: el analítico - la cantidad de población privada de cierto bien o capacidad-, el estructural - la vinculación de cierta carencia con otras carencias relevantes- y el causal - el grado explicativo de cada privación en el surgimiento o disminución de la pobreza-. 